\section{TARMED: la boîte noire qui n'en est pas une}

\author{
Groupe de travail TARMED FMH
}

Le TARMED, cette mécanique tarifaire de mauvais augure que certains appellent «boîte noire», donne lieu à de nombreuses discussions. On se pose des questions sur le fonctionnement de cette machine complexe et la façon dont ses différents éléments y participent, mais on aimerait également connaître le combustible servant à la mettre en marche. Cet article a pour but d'éclairer quelques-uns de ces aspects et d'apporter une vision simplifiée du TARMED, vision qui, pour les connaisseurs de la mécanique tarifaire, manquera peut-être quelque peu de précision.

\section{Les différents univers de la mécanique tarifaire}

Le TARMED, la banque de données tarifaire relationnelle par excellence, dans laquelle tous les éléments sont liés les uns aux autres et pourtant ne dépendent de rien, est principalement constituée de 3 univers: celui du GRAT pour le calcul des prestations médicales (PM) et des prestations techniques (PT) au cabinet médical, l'univers INFRA pour le calcul des PT à l'hôpital ainsi que l'univers de la banque de données TARMED, qui a donné lieu à la création du navigateur TARMED, bien connu de tous.

\section{L'univers GRAT (cabinet médical)}

Le TARMED est caractérisé par le fractionnement de chaque position tarifaire en une prestation médicale (PM) et une prestation technique (PT).

\section{Le calcul de la PT du GRAT}

Dans le cadre de l'étude permanente des coûts des cabinets médicaux (RoKo), des questionnaires ont été envoyés à des médecins en pratique privée, en les priant de fournir des données quant aux paramètres suivants:

- les diverses dépenses (p. ex. frais pour les locaux, frais de personnel, médicaments, frais pour la formation continue, etc.);

- la superficie des locaux (p. ex. salle de consultation, laboratoire, pharmacie, etc.).

Les données récoltées sont "passées au crible», ce qui signifie que l'on vérifie si celles-ci répondent aux critères GRAT définis par la direction du projet TARMED (p. ex. âge du médecin, exploitation du cabinet depuis au moins 3 ans, activité minimale en pratique privée, etc.). Ensuite on calcule, à partir des données ainsi filtrées, la moyenne arithmétique afin de ga- rantir l'anonymat. Finalement, les données ainsi agrégées sont introduites dans l'univers GRAT et traitées par le système KoReg.

Les étapes suivantes correspondent aux opérations habituelles d'une comptabilité analytique. En abrégé, les coûts d'un cabinet médical, c.-à-d. les types de coûts, sont répartis entre les centres de charges (dans l'univers GRAT: les unités fonctionnelles) qui sont à l'origine de ces frais. En d'autres termes: un médecin appliquant des principes de gestion d'entreprise s'intéressera par exemple à connaître le coût annuel de sa salle de consultation pour pouvoir calculer le revenu qu'il doit générer afin d'assurer une certaine rentabilité, autrement dit, d'être au moins en mesure de couvrir les frais.

Puisque, selon le TARMED, les prestations fournies sont indemnisées par minute, les frais imputés aux unités fonctionnelles, donc dans ce cas à la salle de consultation, doivent être divisés par le nombre de minutes par année où celle-ci est utilisée. Il en résulte ce qu'on appelle le taux de coûts PT GRAT ou, plus simplement, le nombre de francs par minute accordés afin que les frais de l'unité fonctionnelle «salle de consultation" soient couverts à la fin de l'année.

Les données dont on se sert pour le moment sont en grande partie empiriques, c.-à-d. qu'elles sont obtenues à l'aide d'enquêtes.

Les "TARMEDiens» endurcis ne cessent de parler des discussions sans fin soulevées par le minutage de certaines prestations. La durée pendant laquelle une infrastructure est mise à contribution pour une certaine prestation (minutage PT GRAT) a été établie en se fondant sur l'avis d'experts des sociétés de discipline médicale; autrement dit, cette valeur a été définie de façon normative. Les éléments du minutage PT GRAT sont les suivants:

- le temps d'occupation du local, c.-à-d. la durée pendant laquelle le local est utilisé pour fournir la prestation;

- le temps d'attente, c.-à-d. le temps nécessaire pour remettre en état l'infrastructure entre deux consultations.

En multipliant le taux de coûts PT GRAT par le minutage PT GRAT, on obtient la PT de la position tarifaire, c.-à-d. de la prestation fournie au cabinet médical.

\section{Calcul PM}

Là également, la base adoptée dans le TARMED étant la minute, l'indemnisation de la prestation médicale est fondée sur une rémunération non pas horaire, mais par minute (expression technique: taux de coûts PM) qui, multiplié avec le minutage PM GRAT d'une prestation, donne la PM.

Les paramètres suivants permettent de calculer la rémunération par minute (taux de coûts $\mathrm{PM}$ ):

- le revenu de référence,

- le temps de travail annuel,

- la productivité,

- la valeur intrinsèque quantitative,

- l'assistance. 


\section{a) Revenu de référence}

L'étude sur le revenu intitulée "Referenzgrösse zur Bewertung der Ärztlichen Leistung (AL) im Rahmen des GRAT-Tarifs" (1998, Popp) constitue la source principale d'informations pour le calcul du revenu de référence.

Pour estimer le revenu de référence, on s'est référé au principe d'opportunité au sens strict; autrement dit, on s'est basé en premier lieu sur les traitements des médecins d'hôpitaux en position dirigeante.

Pour les calculs de plausibilité, des données supplémentaires ont été rassemblées concernant les revenus ayant cours dans d'autres professions exigeant une formation universitaire.

Malheureusement, probablement en raison des moyens dont dispose la presse pour exploiter un sujet, le revenu de référence est vite devenu le jouet favori du monde politique (le surveillant des prix vous salue bien) et a été réduit de 217000 francs à l'origine à 207000 francs, à cause d'interventions politiques, notamment d'une intervention au niveau de la variable de pilotage.

\section{b) Temps de travail annuel}

Sont déduits des 365 jours de l'année pendant lesquels le médecin peut fournir des prestations: les jours fériés, les vacances, le service militaire, la maladie, la formation continue, etc. On obtient ainsi le temps de travail annuel brut en jours. Celui-ci a principalement été fixé de manière normative, en se référant aux résultats de l'enquête FMH sur le temps de travail dans les cabinets médicaux. Le nombre de jours est multiplié par le nombre moyen d'heures de travail/jour et ce produit est ensuite converti en minutes.

La valeur initiale du temps de travail annuel brut était de 1976 heures (ou 118560 minutes). Mais suite à plusieurs interventions, fondées sur la prise de position du surveillant des prix, ce chiffre a également été revu à la baisse; il est actuellement de 1920 heures (ou 115200 minutes).

\section{c) Productivité}

Lors des séances de la Conférence des présidents et de la Chambre médicale, les chiffres concernant la productivité n'ont cessé d'être critiqués, soit parce qu'ils étaient trop hauts ou alors trop bas. Mais que signifie en fait le terme productivité?

Comme pour toute autre profession, le médecin ne peut pas toujours être occupé à des tâches prises en compte dans le tarif; il ne travaille pas toujours auprès du patient ou pour lui. Le détenteur du cabinet médical doit consacrer un certain temps à des tâches de gestion (prestations "overhead") pour lesquelles il n'existe pas de position tarifaire dans le TARMED. Ce temps est en revanche déduit du temps de travail annuel brut.

La productivité, c'est-à-dire la part du temps de travail donnant lieu à tarification au temps de travail annuel brut, a été déterminée au moyen de l'enquête FMH sur le temps de travail dans les cabinets médicaux et des discussions d'experts GRAT. En raison de la complexité inégale des prestations fournies, la productivité a été établie séparément pour chaque unité fonctionnelle.

\section{d) Valeur intrinsèque quantitative}

Chaque position tarifaire se voit attribuer une valeur intrinsèque qualitative et une valeur intrinsèque quantitative. En d'autres termes: pour chaque position tarifaire, on détermine quelle qualification professionnelle (titre de spécialiste, formation approfondie, attestation de formation complémentaire ou certificat d'aptitude technique), c'est-à-dire de quelle valeur intrinsèque qualitative un médecin doit disposer pour pouvoir facturer cette prestation à la charge de l'assurance sociale.

La valeur intrinsèque quantitative est un facteur de calcul. En principe, les divers niveaux (FMH 5, 6, ... 12) représentent le nombre d'années de formation postgraduée qu'un médecin doit accomplir pour pouvoir fournir une prestation d'une qualité indiscutable. Les diverses valeurs intrinsèques sont quantifiées en leur assignant un facteur.

Le taux de cô̂ts PM (ou rémunération par minute) est donc le produit de a) et d) divisé par le produit de b) et c).

\section{e) Assistance}

Une distinction a été introduite entre la $1^{\text {re }}$ et la $2^{\mathrm{e}}$ assistance. Le $1^{\text {er }}$ assistant est mis au même niveau que l'opérateur en ce qui concerne la productivité et la valeur intrinsèque quantitative. En revanche, le $2^{\mathrm{e}}$ assistant se voit attribuer un taux de productivité et un niveau de valeur intrinsèque fixes. La rémunération par minute $\mathrm{du} 1^{\mathrm{er}}$ assistant dépend de la valeur intrinsèque quantitative, alors que le $2^{\mathrm{e}}$ assistant est indemnisé selon un taux fixe.

Concernant l'assistance, l'indication du nombre d'assistants en chiffre décimal a donné lieu à de nombreuses discussions et notamment à la question de savoir comment répartir ces places "décimales» entre assistants dans la réalité. La solution est simple. Ces chiffres correspondent à une fréquence moyenne et doivent être interprétés de la façon suivante: $1.8=$ un assistant est nécessaire dans 100\% des cas et, dans $80 \%$ des cas, on a besoin d'un $2^{\mathrm{e}}$ assistant.

Le taux de coûts PM de l'assistant se calcule de façon analogue à celui de l'opérateur, tout en prenant en considération l'échelonnement quant à la rémunération par minute.

Le minutage PM GRAT a, lui aussi, été déterminé sur la base des discussions d'experts, c.-à-d. de façon normative pour chaque position tarifaire; il se compose des éléments suivants:

- prestation au sens strict, bien connue désormais dans le milieu TARMED, sous l'appellation «temps incision-suture";

- temps de préparation et de finition (terme également bien connu chez les personnes intéressées par le TARMED);

- temps consacré à la rédaction des constatations médicales. 
Comme pour la PT, la PM est le produit du taux de coûts PM (rémunération par minute) par le minutage PM GRAT.

\section{SCSI}

Le 2 février 2000, la Chambre médicale a décidé de rehausser d'un facteur 1.2 les prestations opératoires et invasives. Cette décision a été mise en application dans la structure tarifaire du TARMED, en introduisant un indice de gravité et de complexité (SeverityComplexity-Score-Index, SCSI) global, si bien que toutes les PM concernées sont multipliées par ce SCSI.

\section{Univers INFRA (hôpital)}

L'univers INFRA, contrairement à celui du GRAT, n'est pas fondé sur des chiffres empiriques mais sur des valeurs-clés fixées de manière normative pour une unité fonctionnelle. Le caractère économique et l'état idéal (optimalisation sur le plan de l'organisation, du rendement, du personnel, de l'aménagement du bâtiment et de son exploitation, et des installations) sont prioritaires pour INFRA. Par opposition à l'univers GRAT, ce n'est pas la répartition des données relatives aux coûts entre les différentes unités fonctionnelles qui est important, mais bien la détermination des valeurs-clés centrales fondées sur l'expérience.

Les domaines fonctionnels de l'univers INFRA sont les suivants:

- domaine des soins,

- domaine des examens et des traitements,

- gestion et domaine général,

- approvisionnement et recyclage,

- domaine périphérique.

Analogue au taux de coûts PT GRAT, le taux de coûts PT INFRA est le produit des deux variables coûts et temps et est indiqué en Fr./minute.

La variable des coûts

Les coûts se calculent à partir des frais de personnel non médical, des frais d'exploitation des installations et des frais matériels et redevances.

\section{a) Frais du personnel non médical}

Sur la base des valeurs-clés (salaires, prestations sociales, temps de travail normal, supplément pour dérangement, jours de travail productifs, temps de travail productif et non productif par année), on calcule un taux de coûts en $\mathrm{Fr}$./minute; celui-ci répond à la question de savoir combien coûte par minute le personnel non-médical travaillant de manière directe ou indirecte pour cette unité fonctionnelle (p. ex. personnel auxiliaire pour la préparation des appareils). b) Frais d'exploitation des installations

Partant de la surface exploitable nette (surface qui peut effectivement être utilisée) d'une unité fonctionnelle, un calcul à plusieurs niveaux permet d'estimer, pour une année, les frais d'investissements immobiliers (les frais d'investissement de l'unité fonctionnelle), les frais d'investissement construction (frais d'appareils inclus), les frais d'exploitation des installations (y compris les amortissements, l'intérêt hypothécaire, etc.). Si l'on divise ces frais d'exploitation des installations par le produit entre les jours d'exploitation par année et le nombre d'heures d'exploitation par jour, le résultat obtenu est le taux des frais d'exploitation des installations/minute.

\section{c) Frais matériels et redevances}

En fait, le point essentiel ici est celui de la répartition de la quote-part des tâches de gestion (overhead) entre les unités fonctionnelles. Ce pourcentage a été évalué de manière purement normative et se situe en règle générale entre 65 et $70 \%$, ce qui signifie que, selon les cas de figure, jusqu'à 65 ou 70\% des frais overhead peuvent être reportés sur les unités fonctionnelles.

\section{La variable du temps}

Le calcul du taux de coûts PT INFRA ne se base pas seulement sur les frais réels d'une unité fonctionnelle, mais également sur les temps productifs et non productifs ou, plus précisément, les temps productifs et non productifs du personnel et des installations de l'unité fonctionnelle. Ces valeurs sont établies à partir d'un temps de travail fixé comme norme ou d'un temps d'exploitation par année, en tenant compte de la productivité du personnel et de l'exploitation des installations.

En multipliant le taux de coûts PT INFRA par le minutage PT INFRA (composé du temps d'occupation du local et du temps d'attente et fixé individuellement pour chaque prestation), on obtient la $P T$ pour les prestations fournies dans le secteur hospitalier et donc calculées selon le modèle INFRA.

\section{Univers de la banque de données TARMED}

Les PM et les PT calculées dans les univers GRAT et INFRA sont saisies dans la banque de données TARMED, où la nomenclature tarifaire (désignation de la prestation, numéros de position, interprétations, etc.) est régulièrement mise à jour. Ce n'est que par la réunion de tous ces éléments en un seul lieu que le navigateur TARMED peut jouer son rôle.

L'externalisation du calculateur s'accompagne d'un risque d'erreur considérable. C'est pour cette raison que, dans le cadre du "redesign" (consolidation de la mécanique tarifaire), les banques de données GRAT, INFRA et TARMED seront réunies en un seul endroit pour être gérées de façon centralisée. 